\title{
Weighted Least Squares Solution for RSS based Localization in Correlated Shadowing
}

\author{
Zeyuan Li, Pei-Jung Chung \\ Institute for Digital Communications, University of Edinburgh, EH9 3JL \\ Email: z.li@ed.ac.uk, peijung.chung@gmail.com
}

\begin{abstract}
In this paper, we study the received signal strength (RSS) based localization problem with correlated shadowing between pairs of RSS measurements. By linearizing the correlated RSS model, a weighted least squares (WLS) is formulated to obtain the target location. We also study the correlated shadowing when differential received signal strength (DRSS) is deployed as measurements. Numerical simulations show that the proposed algorithms outperform the algorithms that do not take the correlation between measurements into consideration.
\end{abstract}

\section{INTRODUCTION}

Node localization of sensor nodes in wireless sensor networks (WSN) has become an important task due to the emerging location based services, such as surveillance, controlling and tracking [1]. Thus, many researchers have focused on WSN positioning systems. Most WSN positioning systems are accomplished through RSS, angle of arrival (AoA) or time of arrival (ToA) measurements. Compared with AoA and ToA, RSS is a low complexity, hardware cost effective solution.

In most RSS based positioning systems, the transmitter power and path loss exponent are known in advance. However, sometimes it is difficult to attain these environment factors. Therefore, it is popular to set transmitter power as an unknown parameter. One way to cancel the transmitter power is to subtract RSS measurement with each other, called DRSS [2].

As we know, RSS based localization in the context of ML is non-linear. In order to solve this problem, several approaches have been proposed, including the well known methods, such as maximum likelihood (ML), semidefinite programming (SDP) [3] and linear least squares (LLS) [4]. The ML and SDP methods provide very good performance with a significantly higher computational cost, while the LLS method is computationally efficient.

The majority of existing studies in RSS model localization simply assume that shadowing noise at two positions are independent. In practice, shadowing noise is correlated due to similar topography among RSS propagation paths. And the correlation coefficient is mainly affected by frequency, direction travelled and topography [2]. Hence, it is important to take correlation into consideration when using RSS measurements. In this paper, we propose a WLS algorithm for both RSS and DRSS measurements with correlated shadowing. The proposed algorithm has consists of two stages. In the first stage, a WLS is formed by linearizing RSS model to compute the source location along with an auxiliary variable. In the second stage, we improve the performance by using the relationship between source location and the auxiliary varible [4], [5].

The rest of the paper is organized as follows. In Section II, the system model is described. Section III presents the proposed WLS for correlated RSS measurements and Section IV presents the proposed WLS for correlated DRSS measurements. In Section V, we discuss the simulation results. Concluding remarks are given in Section VI.

\section{Measurement Model}

Assume that there is a 2-dimensional WSN which has a target node with unknown coordinates denoted by $\mathbf{x}_{s}=[x, y]^{T}$ to be estimated. We also assume that the WSN locates the target node using $M$ anchor nodes with known positions denoted by $\mathbf{s}_{i}=\left[x_{i}, y_{i}\right]^{T}$ for $i=1, \ldots, M$. The path loss $P_{i}$ (in $\left.\mathrm{dB}\right)$ under log-normal shadowing is modeled as [6]

$$
P_{i}=P_{0}-10 \alpha \log _{10} \frac{d_{i}}{d_{0}}+n_{i} \quad i=1, \ldots, M
$$

where $P_{0}$ is the path loss measured at a reference distance $d_{0}, d_{i}$ is the distance between $\mathbf{x}_{s}$ and $\mathbf{x}_{i}, \alpha$ is the path loss exponent which varies between 2 and 5 depending on the environment [6], and $n_{i}$ (also in $\mathrm{dB}$ ) represents the log-normal shadowing noise.

In this paper, we assume that the shadowing noise is not independent. It has been found in real scenario experiments that the correlation coefficient varies from 0.2 to 0.8 for indoor environments [7] and 0.2 to 0.5 for outdoor environments [8]. Thus, it is very important to take the correlation into account for RSS based location estimation. Similar to [2], [3], [7], the covariance matrix of $n_{i}$ is assumed to be

$$
\left[\mathbf{Q}_{n}\right]_{i j}=\left\{\begin{array}{ll}
\sigma_{i}^{2} & \text { if } i=j \\
\rho_{i j} \sigma_{i} \sigma_{j} & \text { if } i \neq j
\end{array},\right.
$$

where $\sigma_{i}$ is the standard deviation of shadowing noise, $\rho_{i j}$ is the correlation coefficient between the $i$ th and $j$ th RSS measurements. The reader may refer to [7] for details of how to calculate $\rho_{i j}$. However, in practice, $\rho_{i j}$ may be difficult to determine. We will test the impact of inaccurate $\rho_{i j}$ on the performance in Section V. In the following, we assume $d_{0}=1 m$, and $P_{0}, \alpha, \sigma_{i}, \rho_{i j}$ are known to the system. 


\section{Weighted LeAst SQUARES APPROACH}

In this section, the proposed WLS estimator for RSS measurements with correlated shadowing will be derived. (1) can be expressed as

$$
l_{i}=-\alpha \ln \left(d_{i}\right)+w_{i},
$$

where $l_{i}=0.1 \ln (10)\left(P_{i}-P_{0}\right)$ and $w_{i}=0.1 \ln (10) n_{i}$ with covariance matrix $\mathbf{Q}_{w}=0.01(\ln (10))^{2} \mathbf{Q}_{n}$. It is evident from (3), it forms a set of non linear equations which are normally solved using iterative methods. However, the equations can also be linearized using WLS.

Let $\hat{l}_{i}=l_{i}-w_{i}$, from (3), we have

$$
d_{i}^{2}=\exp \left(-\frac{2 \hat{l}_{i}}{\alpha}\right)
$$

To linearize (4), we expand $d_{i}^{2}$ using $d_{i}^{2}=\left(x-x_{i}\right)^{2}+(y-$ $\left.y_{i}\right)^{2}$ and form it in matrix

$$
\mathbf{A}_{1} \boldsymbol{\theta}_{1}=\mathbf{b}_{1}
$$

where

$$
\mathbf{A}_{1}=\left[\begin{array}{ccc}
-2 x_{1} & -2 y_{1} & 1 \\
\vdots & \vdots & \vdots \\
-2 x_{M} & -2 y_{M} & 1
\end{array}\right], \quad \boldsymbol{\theta}_{1}=\left[\begin{array}{c}
x \\
y \\
x^{2}+y^{2}
\end{array}\right]
$$

and

$$
\mathbf{b}_{1}=\left[\begin{array}{c}
\exp \left(-\frac{2 \hat{l}_{1}}{\alpha}\right)-x_{1}^{2}-y_{1}^{2} \\
\vdots \\
\exp \left(-\frac{2 \hat{l}_{M}}{\alpha}\right)-x_{M}^{2}-y_{M}^{2}
\end{array}\right]
$$

In [9], an unbiased distance estimator under independent log-normal shadowing is presented. Applying the method suggested in [9], the distance estimator $\hat{d}_{i}^{2}$ is obtained as

$$
\hat{d}_{i}^{2}=\exp \left(-\frac{2 l_{i}}{\alpha}-\frac{2 \eta_{i}^{2}}{\alpha^{2}}\right)
$$

where $\eta_{i}^{2}=0.01(\ln (10))^{2} \sigma_{i}^{2}$.

However, the corresponding covariance matrix in [9] of $\hat{d}_{i}^{2}$ only works when each pair of RSS link is independent. Here we extend their work to the correlated case. According to [9], $\hat{d}_{i}^{2}$ can also be expressed as

$$
\hat{d}_{i}^{2}=d_{i}^{2} \exp \left(-\frac{2 w_{i}}{\alpha}-\frac{2 \eta_{i}^{2}}{\alpha^{2}}\right)
$$

Then the covariance matrix for $\hat{d}_{i}{ }^{2}$ denoted by $\mathbf{Q}_{c}$ is given by

$$
\begin{aligned}
& \left.\mathbf{Q}_{c}\right]_{i j}=E\left[\left(\hat{d}_{i}^{2}-d_{i}^{2}\right)\left(\hat{d}_{j}^{2}-d_{j}^{2}\right)\right] \\
= & E\left[d_{i}^{2} d_{j}^{2}\left(\exp \left(-\frac{2 w_{i}}{\alpha}-\frac{2 \eta_{i}^{2}}{\alpha^{2}}\right)-1\right)\right. \\
& \left.\left(\exp \left(-\frac{2 w_{j}}{\alpha}-\frac{2 \eta_{j}^{2}}{\alpha}\right)-1\right)\right] \\
= & d_{i}^{2} d_{j}^{2}\left\{\exp \left(-\frac{2\left(\eta_{i}^{2}+\eta_{j}^{2}\right)}{\alpha^{2}}\right) E\left[\exp \left(-\frac{2\left(w_{i}+w_{j}\right)}{\alpha}\right)\right]\right. \\
& -\exp \left(-\frac{2 \eta_{j}^{2}}{\alpha^{2}}\right) E\left[\exp \left(-\frac{2 w_{j}}{\alpha}\right)\right] \\
& \left.-\exp \left(-\frac{2 \eta_{i}^{2}}{\alpha^{2}}\right) E\left[\exp \left(-\frac{2 w_{i}}{\alpha}\right)\right]+1\right\} \\
= & d_{i}^{2} d_{j}^{2}\left\{\exp \left(-\frac{2\left(\eta_{i}^{2}+\eta_{j}^{2}\right)}{\alpha^{2}}\right) E\left[\exp \left(-\frac{2\left(w_{i}+w_{j}\right)}{\alpha}\right)\right]-1\right\} .
\end{aligned}
$$

If $i=j$, (8) can be written as

$$
\left[\mathbf{Q}_{c}\right]_{i i}=d_{i}^{4}\left(\exp \left(\frac{4 \eta_{i}^{2}}{\alpha^{2}}\right)-1\right) .
$$

If $i \neq j$, from [10], we can have

$$
\begin{aligned}
& E\left[\exp \left(-\frac{2\left(w_{i}+w_{j}\right)}{\alpha}\right)\right]=\exp \left[\frac{2}{\alpha^{2}} \operatorname{Var}\left(w_{i}+w_{j}\right)\right] \\
& =\exp \left[\frac{2}{\alpha^{2}}\left(\operatorname{Var}\left(w_{i}\right)+\operatorname{Var}\left(w_{j}\right)+2 \operatorname{Cov}\left(w_{i}, w_{j}\right)\right)\right] \\
& =\exp \left[\frac{2}{\alpha^{2}}\left(\eta_{i}^{2}+\eta_{j}^{2}+2 \rho_{i j} \eta_{i} \eta_{j}\right)\right] .
\end{aligned}
$$

Substitute (9) and (10) into (8), we can have

$$
\left[\mathbf{Q}_{c}\right]_{i j}=\left\{\begin{array}{ll}
d_{i}^{4}\left(\exp \left(\frac{4 \eta_{i}^{2}}{\alpha^{2}}\right)-1\right) & \text { if } i=j \\
d_{i}^{2} d_{j}^{2}\left(\exp \left(\frac{4 \rho_{i j} \eta_{i} \eta_{j}}{\alpha^{2}}\right)-1\right) & \text { if } i \neq j
\end{array} .\right.
$$

In practice, $d_{i}^{2}$ in $\mathbf{Q}_{c}$ is replaced with $\hat{d}_{i}^{2}$.

The WLS solution of $\boldsymbol{\theta}_{1}$ is then given by [11]

$$
\hat{\boldsymbol{\theta}}_{1}=\left(\mathbf{A}_{1}^{T} \mathbf{Q}_{c} \mathbf{A}_{1}\right)^{-1} \mathbf{A}_{1}^{T} \mathbf{Q}_{c} \mathbf{A}_{1} .
$$

The position $\mathbf{x}_{s}$ to be estimated is the first and second elements of $\hat{\boldsymbol{\theta}}_{1}$.

From the WLS theory [11], $\hat{\boldsymbol{\theta}}_{1}$ is unbiased if elements in $\hat{\boldsymbol{\theta}}_{1}$ are independent. The covariance of $\hat{\boldsymbol{\theta}}_{1}$ is

$$
\operatorname{cov}\left(\hat{\boldsymbol{\theta}}_{1}\right)=\left(\mathbf{A}_{1}^{T} \mathbf{Q}_{c} \mathbf{A}_{1}\right)^{-1} .
$$

As the elements in $\hat{\boldsymbol{\theta}}_{1}$ (12) are not independent, we can make use their relationship to form a second WLS to improve the performance. Let $\boldsymbol{\theta}_{1}(i)$ represent the $i$ th element of vector $\boldsymbol{\theta}_{1}$. Following [4], we can construct another set of equations

$$
\varepsilon=\mathbf{b}_{2}-\mathbf{A}_{2} \boldsymbol{\theta}_{2}
$$

where

$$
\mathbf{A}_{2}=\left[\begin{array}{ll}
1 & 0 \\
0 & 1 \\
1 & 1
\end{array}\right], \quad \boldsymbol{\theta}_{2}=\left[\begin{array}{l}
x^{2} \\
y^{2}
\end{array}\right]
$$


where

$$
\mathbf{b}_{2}=\left[\begin{array}{c}
\hat{\boldsymbol{\theta}}_{1}^{2}(1) \\
\hat{\boldsymbol{\theta}}_{1}^{2}(2) \\
\hat{\boldsymbol{\theta}}_{1}(3)
\end{array}\right] .
$$

$\boldsymbol{\varepsilon}$ is the perturbation term derived from (14). When $\hat{\boldsymbol{\theta}}_{1}(1)$ is sufficiently close to $x$,we have

$\hat{\boldsymbol{\theta}}_{1}^{2}(1)-x^{2}=\left(\hat{\boldsymbol{\theta}}_{1}(1)+x\right)\left(\hat{\boldsymbol{\theta}}_{1}(1)-x\right) \approx 2 x\left(\hat{\boldsymbol{\theta}}_{1}(1)-x\right)$.

Similarly, we have

$$
\hat{\boldsymbol{\theta}}_{1}^{2}(2)-y^{2} \approx 2 y\left(\hat{\boldsymbol{\theta}}_{1}(2)-y\right) .
$$

Then $\varepsilon$ is given by

$$
\boldsymbol{\varepsilon}=\left[2 x\left(\hat{\boldsymbol{\theta}}_{1}(1)-x\right), 2 y\left(\hat{\boldsymbol{\theta}}_{1}(2)-y\right), \hat{\boldsymbol{\theta}}_{1}(3)-x^{2}-y^{2}\right]^{T} .
$$

Employing (13), we obtain the covariance of $\varepsilon$, denoted by $\mathbf{Q}_{r}$, as follows

$$
\mathbf{Q}_{r}=\operatorname{diag}(2 x, 2 y, 1) \operatorname{cov}\left(\hat{\boldsymbol{\theta}}_{1}\right) \operatorname{diag}(2 x, 2 y, 1) .
$$

The unknown $x$ and $y$ is replaced with $\hat{\boldsymbol{\theta}}_{1}(1)$ and $\hat{\boldsymbol{\theta}}_{1}(1)$ in practice.

Finally, the solution of (14) is given by

$$
\hat{\boldsymbol{\theta}}_{2}=\left(\mathbf{A}_{2}^{T} \mathbf{Q}_{r} \mathbf{A}_{2}\right)^{-1} \mathbf{A}_{2}^{T} \mathbf{Q}_{r} \mathbf{b}_{2} \text {. }
$$

As the sign of $\mathbf{x}_{s}$ should be the same as in (12), the target node location is given by

$$
\mathbf{x}_{R S S}=\mathbf{W}_{1} \sqrt{\hat{\boldsymbol{\theta}}_{2}} .
$$

where $\mathbf{W}_{1}=\operatorname{diag}\left\{\operatorname{sign}\left(\hat{\boldsymbol{\theta}}_{1}(1: 2)\right)\right\}$.

\section{EXTENSION TO DRSS}

The proposed method is also applicable to other positioning measurements such as DRSS. We shall illustrate the use of this approach for DRSS under correlated shadowing noise.

The DRSS is obtained by subtracting pairs of RSS measurements with each other. While there are $M(M-1)$ possible pairs of RSS combinations, the DRSS information can all be obtained from combinations of $M-1$ of them. For simplicity, we only consider the measurements

$$
p_{i 1}=P_{i}-P_{1} \quad i=2, \ldots, M .
$$

Observe that $P_{0}$ is canceled in (20). Let $v_{i 1}=n_{i}-n_{1}$, $q_{i 1}=d_{i} / d_{1}$. According to (1), (20) can be expressed as

$$
p_{i 1}=P_{i}-P_{1}=-10 \alpha \log _{10} q_{i 1}+v_{i 1} \quad i=2, \ldots, M .
$$

Specifically, the covariance matrix $\mathbf{Q}_{v}$ is

$$
\mathbf{Q}_{v}=\mathbf{K} \mathbf{Q}_{w} \mathbf{K}^{T}
$$

$$
\mathbf{K}=\left[\begin{array}{ccccc}
-1 & 1 & 0 & \ldots & 0 \\
-1 & 0 & 1 & \ldots & 0 \\
\vdots & \vdots & \vdots & \ddots & \vdots \\
-1 & 0 & 0 & \ldots & 1
\end{array}\right]
$$

Similar to (3), the observed differential path loss can be written as

$$
\lambda_{i 1}=-\alpha \ln q_{i 1}+e_{i 1},
$$

where $\lambda_{i 1}=0.1 \ln (10)\left(P_{i}-P_{1}\right)$ and $e_{i 1}=0.1 \ln (10) v_{i 1}$ with covariance matrix $\mathbf{Q}_{e}=0.01(\ln (10))^{2} \mathbf{Q}_{v}$.

Applying the same approach in (7) and (11), the unbiased estimator of $q_{i 1}^{2}$ is given by

$$
\hat{q}_{i 1}^{2}=\exp \left(-\frac{2 \lambda_{i 1}}{\alpha}-\frac{2\left[\mathbf{Q}_{e}\right]_{i i}}{\alpha^{2}}\right)
$$

and its covariance matrix $\mathbf{Q}_{q}$ is given by

$$
\left[\mathbf{Q}_{q}\right]_{i j}=\left\{\begin{array}{ll}
\lambda_{i 1}^{4}\left(\exp \left(\frac{4\left[\mathbf{Q}_{e}\right]_{i i}}{\alpha^{2}}\right)-1\right) & \text { if } i=j \\
\lambda_{i 1}^{2} \lambda_{j 1}^{2}\left(\exp \left(\frac{4\left[\mathbf{Q}_{e}\right]_{i j}}{\alpha^{2}}\right)-1\right) & \text { if } i \neq j
\end{array} .\right.
$$

We now develop the WLS solution for this problem. Similar to Section II, it has two stages. The first stage uses $q_{i 1}$ to obtain the estimate of $\mathbf{x}_{s}$ and $d_{1}^{2}$ under the assumption that $\mathbf{x}_{s}$ and $d_{1}^{2}$ are independent. In the second stage, we exploit the relationship between $\mathbf{x}_{s}$ and $d_{1}^{2}$ to improve the performance of the algorithm. This two step stage method has been used previously for energy based localization [5].

As $q_{i 1}^{2} d_{1}^{2}=d_{i}^{2}$, to linearize the equation, substitute $d_{i}^{2}=$ $\left(x-x_{1}\right)^{2}+\left(y-y_{1}\right)^{2}$ into the equation, and write it in matrix form

$$
d_{1}^{2} \Delta \mathbf{q}=\mathbf{h}_{1}-\mathbf{G}_{1} \mathbf{z}_{1}
$$

where

$$
\begin{gathered}
\mathbf{G}_{1}=\left[\begin{array}{ccc}
2\left(x_{1}-x_{2}\right) & 2\left(y_{1}-y_{2}\right) & 1-q_{21}^{2} \\
\vdots & \vdots & \vdots \\
2\left(x_{1}-x_{M}\right) & 2\left(y_{1}-y_{M}\right) & 1-q_{M 1}^{2}
\end{array}\right], \\
\mathbf{z}_{1}=\left[\begin{array}{c}
x \\
y \\
d_{1}^{2}
\end{array}\right], \quad \mathbf{h}_{1}=\left[\begin{array}{c}
x_{1}^{2}+y_{1}^{2}-x_{2}^{2}-y_{2}^{2} \\
\vdots \\
x_{1}^{2}+y_{1}^{2}-x_{M}^{2}-y_{M}^{2}
\end{array}\right]
\end{gathered}
$$

and $\Delta \mathbf{q}=\left[q_{21}^{2}, \ldots, q_{M 1}^{2}\right]^{T}$ is the error given by vector of $q_{i 1}^{2}$. Then the WLS solution of $\mathbf{z}_{1}$ can be expressed as

$$
\hat{\mathbf{z}}_{1}=\left(\mathbf{G}_{1}^{T} \mathbf{Q}_{q}^{-1} \mathbf{G}_{1}\right)^{-1} \mathbf{G}_{1}^{T} \mathbf{Q}_{q}^{-1} \mathbf{h}_{1} .
$$

The covariance matrix of $\hat{\mathbf{z}}_{1}$ can be expressed as

$$
\operatorname{cov}\left(\hat{\mathbf{z}}_{1}\right)=\left(\mathbf{G}_{1}^{T} \mathbf{Q}_{q}^{-1} \mathbf{G}_{1}\right)^{-1}
$$


In the second stage, we exploit the relationship between $\mathbf{x}$ and $d_{1}^{2}$ to improve estimation accuracy. As $\hat{\mathbf{z}}_{1}(1)$ is an estimate of $x$. Hence

$$
\begin{aligned}
\left(x-x_{1}\right)^{2} & =\left(\hat{\mathbf{z}}_{1}(1)-\Delta \hat{\mathbf{z}}_{1}(1)-x_{1}\right)^{2} \\
& \approx\left(\hat{\mathbf{z}}_{1}(1)-x_{1}\right)^{2}-2\left(\hat{\mathbf{z}}_{1}(1)-x_{1}\right) .
\end{aligned}
$$

where the second order error is neglected. Similarly,

$$
\left(y-y_{1}\right)^{2} \approx\left(\hat{\mathbf{z}}_{1}(2)-y_{1}\right)^{2}-2\left(\hat{\mathbf{z}}_{1}(2)-y_{1}\right) .
$$

Then we can construct the second WLS

$$
\mathbf{B} \Delta \mathbf{z}=\mathbf{h}_{2}-\mathbf{G}_{2} \mathbf{z}_{2},
$$

where

$$
\mathbf{z}_{2}=\left[\begin{array}{c}
\left(x-x_{1}\right)^{2} \\
\left(y-y_{1}\right)^{2}
\end{array}\right], \quad \mathbf{h}_{2}=\left[\begin{array}{c}
\left(\hat{\mathbf{z}}_{1}(1)-x_{1}\right)^{2} \\
\left(\hat{\mathbf{z}}_{1}(2)-y_{1}\right)^{2} \\
\hat{\mathbf{z}}_{1}(3)
\end{array}\right]
$$

and

$$
\mathbf{G}_{2}=\left[\begin{array}{ll}
1 & 0 \\
0 & 1 \\
1 & 1
\end{array}\right], \quad \mathbf{B}=\left[\begin{array}{c}
2\left(\hat{\mathbf{z}}_{1}(1)-x_{1}\right) \\
2\left(\hat{\mathbf{z}}_{1}(2)-y_{1}\right) \\
1
\end{array}\right] .
$$

Employing (28), we obtain the covariance of $\mathbf{B} \Delta \mathbf{z}$, denoted by $\mathbf{Q}_{z}$, as follows

$$
\mathbf{Q}_{z}=\mathbf{B}^{-1} \operatorname{cov}\left(\hat{\mathbf{z}}_{1}\right) \mathbf{B}^{-1} .
$$

With the use of (32), the WLS solution is given by

$$
\hat{\mathbf{z}}_{2}=\left(\mathbf{G}_{2}^{T} \mathbf{Q}_{z} \mathbf{G}_{2}\right)^{-1} \mathbf{G}_{2}^{T} \mathbf{Q}_{z} \mathbf{G}_{2} \mathbf{h}_{2} .
$$

According to definition of $\hat{\mathbf{z}}_{1}$ in (26), the target node location is given by

$$
\mathbf{x}_{D R S S}=\mathbf{W}_{2} \sqrt{\hat{\mathbf{z}}_{2}}+\mathbf{s}_{1},
$$

where $\mathbf{W}_{2}=\operatorname{diag}\left\{\operatorname{sign}\left(\hat{\mathbf{z}}_{1}(1: 2)-\mathbf{s}_{1}\right)\right\}$.

\section{Simulation Results}

In this section, we compare the performance of the proposed algorithms through computer simulations. We assume that all the measurements have the same variance and correlation coefficient, i.e. $\sigma_{i}=\sigma, \rho_{i j}=\rho$ [2], [3], [7]. All simulations include 5000 trials. The ML approach is simulated with consideration of correlated shadowing noise. Besides ML, we also compare the proposed algorithms with the LLS [4](labeled as WLS-uncorr), which does not take the correlation among shadowing into consideration. The proposed estimator for correlated shadowing is labeled as WLS-corr in the figures.

In Fig.1, we consider a network using RSS measurement with four anchor nodes at $(0,0),(0,10),(10,0),(10,10)$ and one source to be located at $(7,3)$. Fig. 1 plots the root mean square error (RMSE) of the compared algorithms versus the standard deviation of shadowing. The Cramer-Rao Lower Bound (CRLB) is also included in the figures. The difference between

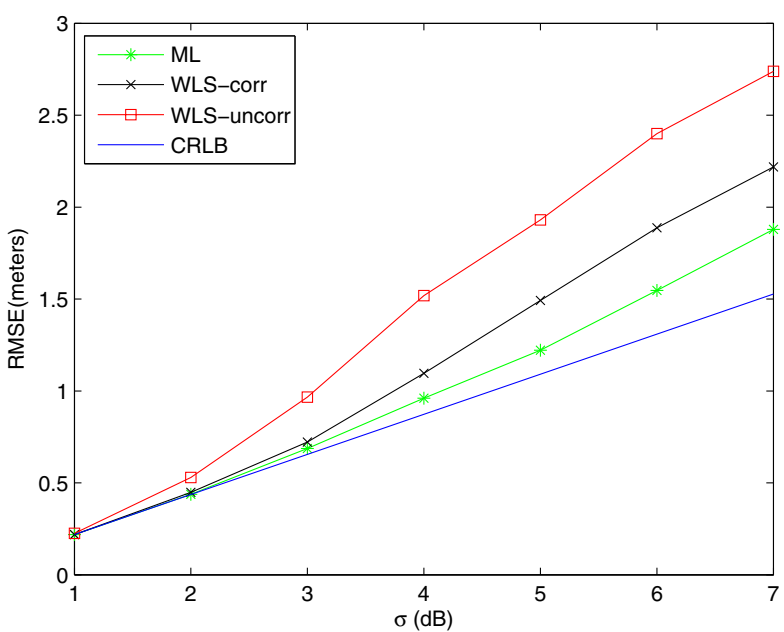

Fig. 1: The RMSE of the estimators versus the standard deviation of shadowing with $\rho=0.7$ and $\alpha=4$ using RSS measurements.

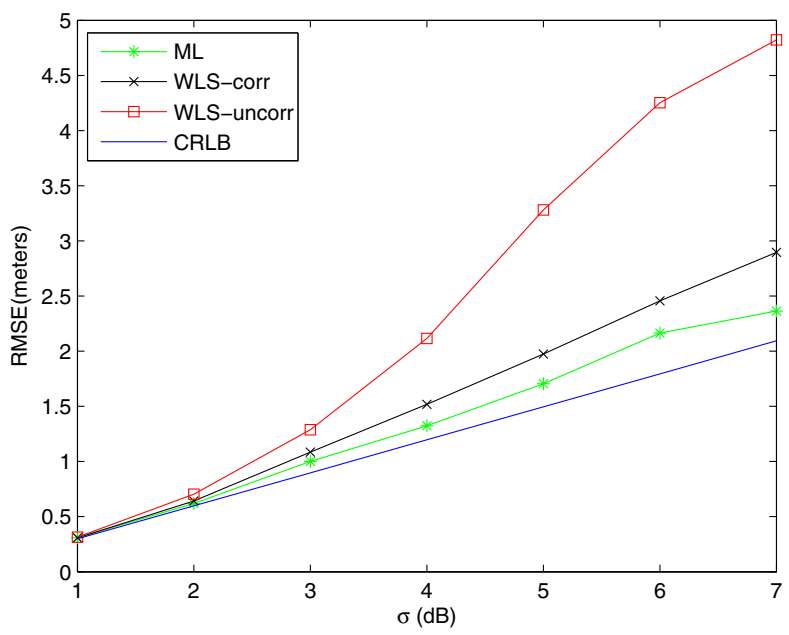

Fig. 2: The RMSE of the estimators versus the standard deviation of shadowing with $\rho=0.8$ and $\alpha=2$ using DRSS measurements.

WLS-corr and WLS-uncorr increases as the standard deviation of the shadowing increases.

In Fig.2, we simulate DRSS measurements with five nodes deployed at $(0,0),(0,10),(10,0),(10,10),(5,5)$. The target to be located is at $(1,4)$. Fig. 2 shows similar results. The proposed approach provides better performance than the WLS-uncorr algorithm which does not consider correlation in shadowing.

Fig.3 shows the RMSEs versus correlation coefficient of shadowing. The nodes configuration is the same as in Fig.1, and $\sigma=3 \mathrm{~dB}$. As can be seen from the plot, under the same shadowing variance, the gap between WLS-corr and WLSuncorr is larger when correlation between RSS measurements increases. Thus, considering correlation among RSS measure- 


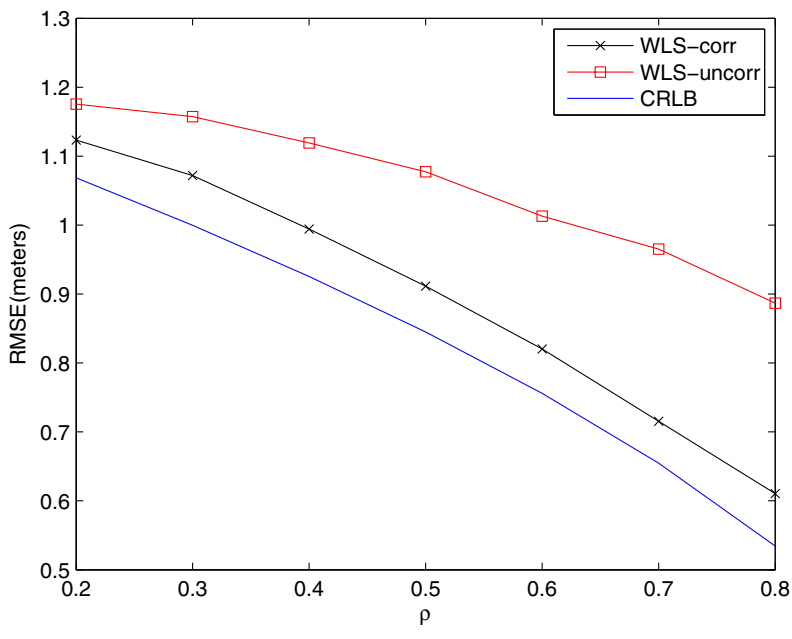

Fig. 3: The RMSE of the estimators versus the correlation coefficient of shadowing with $\sigma=3 \mathrm{~dB}$ and $\alpha=4$ using RSS measurements.

ments in severe shadowing scenario is necessary.

In previous simulations, the correlation coefficient is exactly known to the system. However, an accurate $\rho$ is difficult to calculate based on [7], especially $\rho$ could change due to the change of environment. Now we assume only an imperfect $\hat{\rho}$ is known to the system:

$$
\hat{\rho}=\rho+\delta
$$

where $\delta$ is a zero mean Gaussian random variable with standard variance of 0.2 , which defines the uncertainty of the correlation coefficients. In simulation, $\hat{\rho}$ is constrained between 0.2 to 0.8 , because in reality, the correlation coefficient is between 0.2 to 0.8 [7], [8]. The other parameter configuration is the same as in Fig.1. Please note, the uncertainty parameter $\delta$ is randomly generated in every single run. Fig. 4 shows the RMSE of the proposed estimator with the true and approximate covariance matrix. As can be seen, the performance of ML and WLS-corr both decreased as $\rho$ is inaccurate. However, WLS-corr still outperform WLS-uncorr.

\section{CONCLUSION}

We have proposed a WLS method for RSS and DRSS measurements based localization under correlated shadowing noise. By utilizing the correlation structure of shadowing noise, the proposed algorithms significantly outperform the algorithms that do not take correlation into consideration. Numerical experiments show that the proposed algorithm can achieve comparable results as ML for correlated shadowing. When the correlation coefficient increases, the performance gap between the proposed algorithm and the standard algorithm becomes significant. This observation implies the superiority of our approach in correlated shadowing environments.

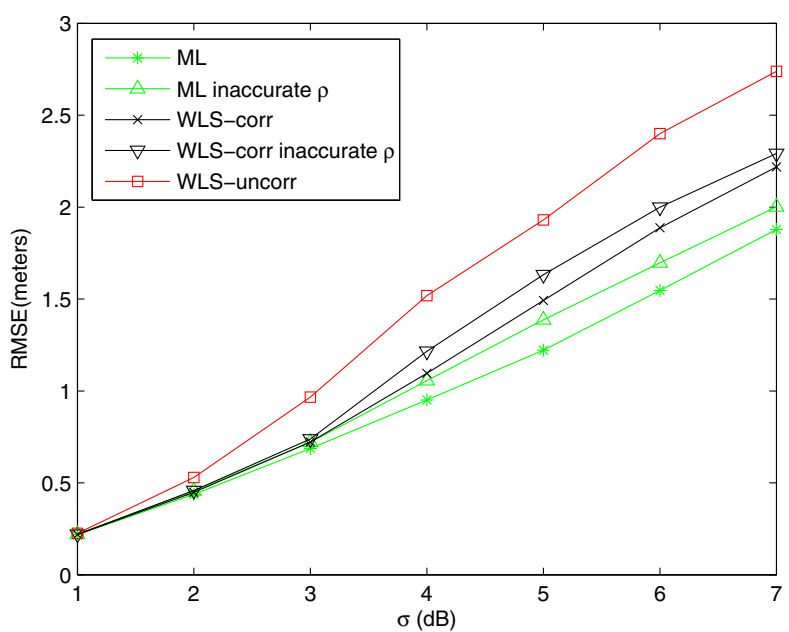

Fig. 4: The RMSE of the estimators with true and inaccurate correlation coefficient.

\section{REFERENCES}

[1] N. Patwari, J. Ash, S. Kyperountas, A. Hero, R. Moses, and N. Correal, "Locating the Nodes: Cooperative localization in wireless sensor networks," Signal Processing Magazine, IEEE, vol. 22, no. 4, pp. 54-69, July 2005.

[2] J. H. Lee and R. Buehrer, "Location Estimation Using Differential RSS with Spatially Correlated Shadowing," in Global Telecommunications Conference, 2009. GLOBECOM 2009. IEEE, Nov 2009, pp. 1-6.

[3] R. Vaghefi and R. Buehrer, "Received Signal Strength-based Sensor Localization in Spatially Correlated shadowing," in Acoustics, Speech and Signal Processing (ICASSP), 2013 IEEE International Conference on, 2013, pp. 4076-4080.

[4] H. C. So and L. Lin, "Linear Least Squares Approach for Accurate Received Signal Strength Based Source Localization,' Signal Processing, IEEE Transactions on, vol. 59, no. 8, pp. 4035-4040, Aug 2011.

[5] K. Ho and M. Sun, "An Accurate Algebraic Closed-Form Solution for Energy-Based Source Localization," Audio, Speech, and Language Processing, IEEE Transactions on, vol. 15, no. 8, pp. 2542-2550, Nov 2007.

[6] T. Rappaport, Wireless Communications: Principles and Practice, 2nd ed. Upper Saddle River, NJ, USA: Prentice Hall PTR, 2001.

[7] J. Liberti and T. Rappaport, "Statistics of Shadowing in Indoor Radio Channels at 900 and $1900 \mathrm{MHz}$," in Military Communications Conference, 1992. MILCOM '92, Conference Record. Communications Fusing Command, Control and Intelligence., IEEE, Oct 1992, pp. 10661070 vol.3.

[8] K. Zayana and B. Guisnet, "Measurements and Modelisation of Shadowing Cross-correlations between two Base-stations," in Universal Personal Communications, 1998. ICUPC '98. IEEE 1998 International Conference on, vol. 1, Oct 1998, pp. 101-105 vol.1.

[9] S. Chitte, S. Dasgupta, and Z. Ding, "Distance Estimation From Received Signal Strength Under Log-Normal Shadowing: Bias and Variance,' Signal Processing Letters, IEEE, vol. 16, no. 3, pp. 216-218, March 2009.

[10] J. A. Gubner, Probability and Random Processes for Electrical and Computer Engineers. New York, NY, USA: Cambridge University Press, 2006.

[11] S. M. Kay, Fundamentals of Statistical Signal Processing: Estimation Theory. Upper Saddle River, NJ, USA: Prentice-Hall, Inc., 1993. 